EISSN: $2706-7947 \quad$ ISSN: $2077-4613$

DOI: 10.36632/mejas/2021.11.4.70

Journal homepage: www.curresweb.com

Pages: 896-909

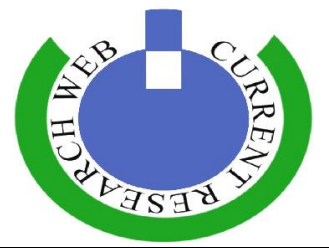

\title{
Evaluation of some locally available casing materials on production of two Agaricus (LANGE) strains
}

\author{
Saad M. Ghazy ${ }^{1}$, Elsayed A. Omer ${ }^{2}$, Ibrahim M. Gomaa ${ }^{1}$ and Mohsen A. El-Mohandes ${ }^{1}$ \\ ${ }^{I}$ Environment and Bio-agriculture Department, Faculty of Agriculture, Al-Azhar University, Cairo, \\ Egypt. \\ ${ }^{2}$ Medicinal and Aromatic plants Research Department, National Research Centre, Giza, Egypt.
}

Received: 07 Oct. $2021 \quad$ Accepted: 06 Nov. $2021 \quad$ Published: 15 Nov. 2021

\begin{abstract}
Six different casing materials were investigated in this study (1) peat moss $(100$, control $),(2)$ peat moss + spent mushroom (3:1), (3) peat moss + vermi-compost (3:1), (4) peat moss + spent mushroom + palm fronds $(2: 1: 1),(5)$ peat moss + palm fronds (1:1) and (6) peat moss + vermicompost + palm fronds (2:1:1), on production and harvest quality of two Agaricus bisporus strains (S-1 and S-2). These experiments were conducted at Tanta Mushroom Station, Gharbia Governorate in 2019. The results showed that the type of casing material had a significant effect on the yield potential and growth behavior of mushroom. Among the tested Casing materials, the best casing layer was the mixture of (peat moss + palm fronds + vermicompost) with both (S-1) and (S-2) strains which exhibited in pinhead initiation (28 and 29.5 days); growth period of the three harvests (42 and 43 days) and total weight (1806.5 and $2542.5 \mathrm{~g}$ ), respectively, compared to control (29 and 30 days, 43 and 43.5 days and 1467.5 and $1507 \mathrm{~g}$, respectively). Total phenolic contents of mushroom insignificantly differed between casing layers with strain (S-1), while it was very clear with strain (S-2) where the casing layer that composed of peat moss + vermin $(3: 1)$ recorded the highest content $(252.93 \mathrm{mg} / 100 \mathrm{~g})$. Flavonoid content was higher in case of peat moss + fronds (1:1) with both strains. The highest antioxidant activity in both strains was achieved when peat moss $100 \%$ was used as acasing layer.
\end{abstract}

Keywords: Agaricus bisporus, casing materials, flavonoid, phenols, antioxidants and yield potential.

\section{Introduction}

The white button mushroom (Agaricus bisporus Lange) is the extensively cultivated and consumed mushroom throughout the world and represented about $56 \%$ of total world mushroom production (Yadav et al., 2017). Mushrooms' market is experiencing a big increase around the world, but the largest expansion is in China, which accounts for more than $85 \%$ of the total worldwide production amounting to 34 million tons produced in 2013; the annual crop income was 3.2 billions dollars worldwide in 2009 and is now more than 4 billion dollars (Sonnenberg et al., 2017). Huge quantities of farm yard manure, vermincompost, saw dust and other organic wastes are generated annually through the activities of agricultural, forest and food processing industries. Egypt is ranked third in the Middle East when it comes to the availability of agriculture palm fronds waste that amounts 71.5 million tons (dried weight) where only $10 \%$ is reused in traditional uses like cages and furniture, while the rest $(90 \%)$ are burned every year. This causes environmental pollution and is a waste of sustainable materials (that include highly recyclable contents, rapidly renewable and biodegradable products) and local resources (Eldeeb 2017). The casing materials of mushroom provide suitable physical, chemical and biological conditions that stimulate initiation of fruiting body formation (Gerrits, 1974). Casing is the part of cultivation of white button mushroom and milky mushroom for fruit body formation. The casing layer is applied after competition of spawn running in mushroom beds. This casing layer prepared from different materials such as loam soil, farm yard manure, vermicompost, sandy soil, cotton waste, saw dust and jute coir pith, for its unique water holding and structural properties. Hence, mushroom cultivation can reduce the

Corresponding Author: Saad M. Ghazy, Environment and Bio-agriculture Department, Faculty of Agriculture, Al-Azhar University, Cairo, Egypt. 
environmental pollution by recycling the agricultural wastes. According tokaur and Rampal,2017), the highest total yield of $A$. bisporus (1066.97 g) was obtained from casing mixture, cocopeat + rice husk + formaline + red soil and the lowest yield $(607.93 \mathrm{~g})$ was obtained with farmyard manure + sandy soil + formaline. The second highest yield $(646.57 \mathrm{~g})$ was obtained from farmyard manure + sandy soil + rice husk + formaline casing mixture. Evaluation of seven different casing mixtures prepared from 5 materials, farm yard manure (FYM), spent compost, vermicompost (VC), coir pith and press mud, for their yield potential and the fruiting body of button mushroom, Agaricus bisporus was carried out by Bhatt et al (2006) and Dhar et al., (2006). They found that FYM, VC were the best casing layers. The good quality of casing soil can be enhanced the yield of mushrooms via quantitative and qualitative attributes of mushroom such as number of fruit bodies, weight, and size of fruiting bodies. Therefore, the present investigation tested some casing materials prepared locally from available wastes for enhancing production of white button mushroom and its effect on some pharmaceutical substances content.

\section{Materials and Methods}

\subsection{Mushroom strains}

Two strains of button mushroom (Agaricus bisporus) were used in this study for growth and yield potential. S-1 strain of the hybrid Sylvan A15 was kindly provided from Microbiological Resources Centre (Cairo Mircen) Ain shams University, kalubia, Egypt whereas S-2 strain was developed by the Environment and Bio-Agriculture team on Petri dishes by hyphal fusion (anastomosis) method. The developed strain was cultured on fresh Potato Dextrose Agar (PDA) medium and maintained by periodic sub-culturing with incubation at $25^{\circ} \mathrm{C}$ for 15 days and kept thereafter in a refrigerator at 2 to $5^{\circ} \mathrm{C}$ for the following studies.

\subsection{Compost preparation}

The compost was prepared by long composting method which took 28-30 days and 8 turning (Mantel et al., 1972). The compost contained wheat straw only $(1000 \mathrm{Kg})$ in addition to required nitrogen supplements as poultry manure $(800 \mathrm{Kg})$; soybean flour $(50 \mathrm{Kg})$ and urea $(6 \mathrm{Kg})$, beside gypsum $(80 \mathrm{Kg})$ to aid in $\mathrm{pH}$ control. The straw is thoroughly watered on clean concrete floor for 24 hr. and mixed with fertilizer separately. The substrate prepared was formed into a large heap of 5 feet width and 7 feet height to encourage intense microbial activities. Turning was carried out every 3-4 days after adding water to maintain the moisture around $75 \%$ to keep aerobic conditions. Gypsum is added at the $3^{\text {rd }}$ turning and the compost was ready for spawning after the $8^{\text {th }}$ turning. More turnings, if the compost heap was not free from ammonia were considered until it is completely ammonia free. polyesterin in box (Length $50 \times$ width $30 \times$ Height $10 \mathrm{~cm}$ ) that contained $10 \mathrm{~kg}$ of wheat straw compost were seeded with $75 \mathrm{~g}$ mushroom seeds and inoculated with Pseudomonas putida as a bio-fertilizer at a rate of $15 \mathrm{ml}$ (that contained $1 \times 10^{8} \mathrm{cfu}$ ) and mixed gently in compost under sterilized conditions (Kapoor, 2004).

\subsection{Spawn preparation}

The spawn was prepared using sorghum (Sorghum bicolor) grains. The grains were washed and then soaked in water for 24 hours for maximum absorption of water and then boiled for 30 minutes as excessive moisture was removed using blotting paper. Three replicates of polyethylene bags $(12 \times 25$ $\mathrm{cm}$ ) each contained $500 \mathrm{~g}$ of sorghum grains were separately prepared for each treatment. The grains were supplemented with $4 \%$ gypsum $\left(\mathrm{CaSO}_{4}\right)$ and $2 \%$ lime $\left(\mathrm{CaOH}_{2}\right)$ and then the polyethylene bags were sterilized at $121{ }^{\circ} \mathrm{C}$ for $30 \mathrm{~min}$. Following sterilization, each polyethylene bag was inoculated under sterilized conditions with mycelial disc ( $9 \mathrm{~mm}$ diameter) of either S-1 or S-2 strains. The sealed inoculated bags were incubated at $25^{\circ} \mathrm{C}$ for 20 days (Ishaq et al., 2017).

\subsection{Procedure}

Six different casing materials (tretmints) were prepared in different ratios and used as a coat soil, (1) control, 100\% peat moss ; (2) peat moss + spent mushroom (3:1); (3) peat moss + vermicompost (3:1); (4) peat moss + spent mushroom + vermicompost $(2: 1: 1)$; (5) peat moss + palm fronds $(1: 1)$ and 
(6) peat moss + palm fronds + vermicompost $(2: 1: 1)$. The $\mathrm{pH}$ was adjusted at 7.0 by adding lime stone (30 kg per cubic meter of casing layer). The casing layer is moistened to $60 \%$ and left to rest for 20 days. Then $30 \%$ wood charcoal $(1-2 \mathrm{~cm})$ was added and pasteurized at $62{ }^{\circ} \mathrm{C}$ for 8 hours. The casing layer was spread on the surface of the compost by hand. Each polyesterin box ( $10 \mathrm{Kg}$ capacity) received $250 \mathrm{~g}$ casing material that formed alayer of about $4 \mathrm{~cm}$ thickness. The medium was covered with plastic and incubated for 15 days at $28 \pm 1^{\circ} \mathrm{C}$. Each treatment was treplicated. After colonizing the cover soil, the boxes were randomly arranged in shelves within a greenhouse with a semi-controlled environment, micro-sprinklers with a flow rate of $7 \mathrm{~L} / \mathrm{hr}$ were used to control the relative humidity between $60-90 \%$. The temperature ranged from 20 to $34{ }^{\circ} \mathrm{C}$. White button mushrooms were harvested on the basis of maturity rather than size. In general, button mushroom was harvested at a stage when cap diameter is twice the length of stipe (Kohli, 1984). Mushroom fruits were have a very kept on refrigerator lower shelves unit used to study the different morphological characteristic. Total yield was the sum of $1^{\text {st }}, 2^{\text {nd }}$ and $3^{\text {rd }}$ flushes.

\section{Determination of total phenolics and, flavonoids contents and antioxidant activity \\ I. Extract preparation}

One gram dry matter of mushroom was mixed with $15 \mathrm{ml}$ of $70 \%$ methanol and stored at room temperature. After $48 \mathrm{~h}$, infusions were filtered through Whatman No. 1 filter paper (Singleton et al., 1999 ) as the extract was received in $100 \mathrm{~mL}$ Erlenmeyer Flasks.

\section{Determination of total phenolic content}

Total phenolic content $(\mathrm{mg} / 100 \mathrm{~g})$ of each treatment were determined in the representative dry mushroom samples according to Singleton et al., (1999) where the reaction mixture was prepared by mixing $0.5 \mathrm{ml}$ of methanolic extract, $2.5 \mathrm{ml}$ of $10 \%$ Folin-Ciocalteu's reagent dissolved in water and $2.5 \mathrm{ml}$ of $7.5 \% \mathrm{Na}_{2} \mathrm{CO}_{3}$. Blank was concomitantly prepared by replacing the methanolic extract by methanol. The samples were thereafter incubated at room temperature for $45 \mathrm{~min}$. The absorbance was determined using spectrophotometer at $\lambda \max =765 \mathrm{~nm}$. The samples were prepared in triplicate for each analysis and the mean value of absorbance was obtained. The same procedure was repeated for the standard solution of gallic acid and the calibration line was construed. Based on the measured absorbance, the concentration of phenolics was read $(\mathrm{mg} / \mathrm{ml})$ from the calibration line; then the content of phenolics in the extracts was expressed in terms of gallic acid equivalent ( $\mathrm{mg}$ of GA/g of mushroom).

\section{Determination of flavonoids content}

Flavonoids content $(\mathrm{mg} / 100 \mathrm{~g})$ of each treatment were determined in representative dry mushroom samples according to Quettier et al., (2000) as the reaction mixture was prepared by mixing $1 \mathrm{ml}$ of methanolic extract and $1 \mathrm{ml}$ of $2 \% \mathrm{AlCl}_{3}$ solution dissolved in methanol. The samples were incubated for an hour at room temperature. The absorbance was determined using spectrophotometer at $\lambda \max =415 \mathrm{~nm}$. The samples were prepared in triplicate for each analysis and the mean value of absorbance was obtained. The same procedure was repeated for the standard solution rutin and the calibration line was construed. Based on the measured absorbance, the concentration of flavonoids was $\mathrm{read}(\mathrm{mg} / \mathrm{ml})$ on the calibration line; then, the content of flavonoids in extracts was expressed in terms of rutin equivalent ( $\mathrm{mg}$ of RU/g of mushroom).

\section{Antioxidant activity}

The ability of the plant extract to scavenge 1,1-Diphenyl-2-picrylhydrazyl (DPPH), free radicals was assessed by the standard method (Tekao et al., 1994), adopted with suitable modifications (Kumarasamy et al., 2007) as DPPH was prepared by dissolving $10 \mathrm{mg}$ sample in $100 \mathrm{ml} 70 \%$ methanol. A known volume $(50 \mu 1)$ of the methanolic extract was dissolved in $450 \mu 170 \%$ methanol then mixed with DPPH $(0.5 \mathrm{ml})$. The mixtures were well shaken and then placed in darkness at room temperature for $30 \mathrm{~min}$. The absorbance was recorded at $517 \mathrm{~nm}$. The control samples contained all the reagents except the extract. Methanol was used to zero the spectrophotometer. The inhibition percentage of the DPPH radical was calculated according to the formula:

$\mathrm{I} \%=[(\mathrm{AB}-\mathrm{AS}) / \mathrm{AB}] \times 100$. 
Where $\mathrm{I}=\mathrm{DPPH}$ inhibition $\%, \mathrm{AB}=$ absorbance of control sample and $\mathrm{AS}=$ absorbance of a tested sample at the end of the reaction. Each assay was carried out in triplicate.

\subsection{Statistical analyzed}

The data were analyzed using SPSS version 14 software. A one-way analysis of variance (ANOVA) was used to test for significance of variation in yield and yield attributes of casing media on different flushes. Means were compared using Duncan test, when F-test from ANOVA was significant at $\mathrm{p}<0.05$.

\section{Results and Discussion}

The casing layer influences yield, quality and uniformity of cropping of the button mushroom. Thus, mushroom productivity, size and mass are directly affected by the casing layer. The casing soil is reported to possess certain physical, chemical and microbiological properties having stimulatory role in Agaricus fruiting (Ahlawat 2002). Shrivastava (2012) stated that any organic material with a high water holding capacity is suitable as a casing material, such as peat moss and perlite mixture (Ergun $e t$ al., 2007); farm yard manure, spent mushroom substrate and vermi compost (Choudhary, 2011) and garden loam soil, farm yard manure, waste tea and vermicompost (Kumar et al., 2018).

\subsection{Effect of casing materials on growth period, weight and shelf-life}

Pinhead initiation, growth period of the three harvests, total mushroom weight and shelf-life period of (S-1) and (S-2) strains are presented in Tables (1) and (2), respectively. The present findings indicated that, the best casing layer for (S-1) and (S-2) strains was peat moss + palm fronds + vermicompost which exhibited for pinhead initiation (28 and 29 days); growth period of the $3^{\text {rd }}$ harvests (42.5 and 39 days) and total weight (2466.5 and $2542.5 \mathrm{~g}$ ), respectively, compared to control (29 and 30 days, 43 and 43.5 days and 1467.5 and $1507 \mathrm{~g}$, respectively). As regard to shelf-life period, (S-1) and (S-2) fruits grown on (peat moss + palm fronds + vermicompost) treatment remained valid for a longer time ( 5 and 4.5 days as fresh fruits and 13 and 9 days when refrigerated, respectively) compared to other treatments. The lowest mushroom total weight was recorded when (peat moss + vermi) was used as (S-1) and (S-2) recorded 1375.5 and $1302 \mathrm{~g}$, respectively. Highly significant differences were observed between all treatments in the pinhead initiation, growth period of the three harvests, total weight and shelf-life period of the two strains. Only the differences between treatments for pinhead initiation and growth period of the first harvest of strain (S-1) were was insignificant.

The casing mixture of peat moss + palm fronds + vermicompost produced higher total yields than other treatments due to improving the aeration palm fronds a result of addition to peat moss +vermicompost. Vermicompost has high water holding capacity as its moisture holding capacity is such that it can be watered without sealing off the compost. Carbon dioxide (and other gases), formed in the compost during spawn running and fruiting, must be able to escape through the casing. Effects of high carbon dioxide concentration include production of small caps and elongation of stipe. The low yields recorded in peat moss + vermicompost, may be attributed not only to its inability to hold sufficient moisture but also the rapid rate at which it loses it by infiltration and evaporation. Water instead of just wetting the casing, may have been draining down into the compost thereby causing sogginess, which interferes with mycelia development and performance. Similar results are recorded by Chandra et al., (2014) that revealed that casing mixture CCP + VC + FYM + SD + Sand recorded the highest yield $(320 \mathrm{~g})$ whereas CCP + FYM ( $250 \mathrm{~g})$ showed lowest yield in the harvesting of second flush. The highest total yield (1112.26 g) was obtained from casing mixture, $\mathrm{CCP}+\mathrm{VC}+\mathrm{FYM}+\mathrm{SD}+\mathrm{Sand}$ and lowest yield $(736.67 \mathrm{~g})$ from CCP + FYM. Casing mixture of CCP + FYM + SD recorded second highest yield (1033.67 g). Singh et al., (2000) found that casing mixture prepared using FYM + spent compost (2:1) performed the best giving a yield of Agaricus bisporus in the first and second crop (18.83 kg and $17.21 \mathrm{~kg} /$ quintal compost).

\subsection{Effect of casing materials on total fruits number}

The average maximum total number of mushroom fruits of strain (S-1) was achieved when the treatment covered with peat moss + palm fronds + vermicompost mixture recording 93 fruits while it recorded 70 fruits when the treatment covered with peat moss + vermicompost as presented in Table (3). Number of mushroom fruits in the control treatment was 81.5. The average maximum number of 
Table 1: Effect of casing materials on growth period, weight and shelf life of $A$. bisporus (S-1) strain.

\begin{tabular}{|c|c|c|c|c|c|c|c|c|c|c|}
\hline \multirow{2}{*}{ Casing media } & \multicolumn{4}{|c|}{$\begin{array}{l}\text { Growth period in days after spawning } \\
\qquad(\text { Mean } \pm \text { SE) }\end{array}$} & \multicolumn{3}{|c|}{$\begin{array}{l}\text { Weight (g) } \\
(\text { Mean } \pm \text { SE) }\end{array}$} & \multirow{2}{*}{$\begin{array}{c}\text { Total } \\
\text { Weight } \\
\text { (g) }\end{array}$} & \multicolumn{2}{|c|}{$\begin{array}{l}\text { Shelf life (days) } \\
\quad(\text { Mean } \pm \text { SE) }\end{array}$} \\
\hline & $\begin{array}{l}\text { Pinhead } \\
\text { initiation }\end{array}$ & $\begin{array}{c}1^{\text {st }} \\
\text { Harv. }\end{array}$ & $\begin{array}{c}2^{\text {nd }} \\
\text { Harv. }\end{array}$ & $\begin{array}{c}3^{\text {rd }} \\
\text { Harv. }\end{array}$ & $\begin{array}{c}1^{\text {st }} \\
\text { Harv. }\end{array}$ & $\begin{array}{c}2^{\text {nd }} \\
\text { Harv. }\end{array}$ & $\begin{array}{c}3^{\text {rd }} \\
\text { Harv. }\end{array}$ & & Fresh air & Refrig. \\
\hline $100 \%$ Peat moss (Control) & $\begin{array}{l}29.0 \\
\pm 0.87^{\mathrm{ab}}\end{array}$ & $\begin{array}{l}33.50 \\
\pm 0.87^{\mathrm{a}}\end{array}$ & $\begin{array}{l}36.5 \\
\pm 0.29^{\mathrm{e}}\end{array}$ & $\begin{array}{c}43.0 \\
\pm 0.29^{\mathrm{c}}\end{array}$ & $\begin{array}{l}539.5 \\
\pm 83.42^{\mathrm{b}}\end{array}$ & $\begin{array}{l}503.0 \\
\pm 15.59^{\mathrm{bc}}\end{array}$ & $\begin{array}{l}425.0 \\
\pm 15.59^{\mathrm{e}}\end{array}$ & 1467.5 & $\begin{array}{c}3.00 \\
\pm 0.00^{\mathrm{bcd}}\end{array}$ & $\begin{array}{l}8.50 \\
\pm 0.29^{\mathrm{bc}}\end{array}$ \\
\hline $\begin{array}{c}75 \% \text { Peat moss }+25 \% \text { Spent } \\
\text { mushroom }\end{array}$ & $\begin{aligned} & 30.5 \\
\pm & 0.29^{\mathrm{ab}}\end{aligned}$ & $\begin{array}{l}33.50 \\
\pm 0.29^{\mathrm{a}} \\
\end{array}$ & $\begin{aligned} & 39.5 \\
\pm & 0.29^{\mathrm{ab}}\end{aligned}$ & $\begin{aligned} & 45.5 \\
\pm & 0.29^{\mathrm{ab}}\end{aligned}$ & $\begin{array}{l}537.5 \\
\pm 99.59^{\mathrm{b}} \\
\end{array}$ & $\begin{array}{c}611.0 \\
\pm 45.61^{\mathrm{b}}\end{array}$ & $\begin{array}{l}553.50 \\
\pm 6.64^{\mathrm{cd}}\end{array}$ & 1702.0 & $\begin{array}{l}2.50 \\
\pm 0.87^{\mathrm{cd}}\end{array}$ & $\begin{array}{c}9.50 \\
\pm 0.29^{\mathrm{b}} \\
\end{array}$ \\
\hline $\begin{array}{l}75 \% \text { Peat moss }+25 \% \\
\text { Vermi }\end{array}$ & $\begin{array}{l}30.0 \\
\pm 0.58^{\mathrm{ab}}\end{array}$ & $\begin{array}{c}33.0 \\
\pm 0.58^{\mathrm{a}}\end{array}$ & $\begin{array}{c}38.0 \\
\pm 0.58^{\mathrm{cd}}\end{array}$ & $\begin{array}{l}44.5 \\
\pm 0.29^{\mathrm{b}}\end{array}$ & $\begin{array}{c}379.0 \\
\pm 12.12^{\mathrm{b}}\end{array}$ & $\begin{array}{l}504.0 \\
\pm 34.01^{\mathrm{bc}}\end{array}$ & $\begin{array}{l}492.5 \\
\pm 63.79^{\mathrm{de}}\end{array}$ & 1375.5 & $\begin{array}{c}4.00 \\
\pm 0.58^{\mathrm{abc}}\end{array}$ & $\begin{array}{l}9.50 \\
\pm 0.29^{\mathrm{b}}\end{array}$ \\
\hline $\begin{array}{l}50 \% \text { Peat moss }+25 \% \text { Spent } \\
\text { mushroom }+25 \% \text { Vermi }\end{array}$ & $\begin{array}{l}31.0 \\
\pm 0.58^{\mathrm{a}}\end{array}$ & $\begin{array}{l}33.5 \\
\pm 0.29^{\mathrm{a}}\end{array}$ & $\begin{array}{c}38.5 \\
\pm 0.29^{\mathrm{bc}}\end{array}$ & $\begin{array}{c}45.5 \\
\pm 0.29^{\mathrm{ab}}\end{array}$ & $\begin{array}{c}550.0 \\
\pm 54.85^{\mathrm{b}}\end{array}$ & $\begin{array}{l}526.0 \\
\pm 45.61^{\mathrm{bc}}\end{array}$ & $\begin{array}{c}677.0 \\
\pm 39.25^{\mathrm{b}}\end{array}$ & 1753.0 & $\begin{aligned} & 4.00 \\
\pm & 0.58^{\mathrm{abc}}\end{aligned}$ & $\begin{aligned} & 8.50 \\
\pm & 0.29^{\mathrm{bc}}\end{aligned}$ \\
\hline $\begin{array}{c}50 \% \text { Peat moss }+50 \% \\
\text { fronds }\end{array}$ & $\begin{array}{c}30.0 \\
\pm 0.58^{\mathrm{ab}}\end{array}$ & $\begin{array}{c}33.5 \\
\pm 0.29^{\mathrm{a}}\end{array}$ & $\begin{array}{l}40.0 \\
\pm 0.58^{\mathrm{a}}\end{array}$ & $\begin{array}{c}46.0 \\
\pm 0.58^{\mathrm{a}}\end{array}$ & $\begin{array}{c}733.5 \\
\pm 32.04^{\mathrm{a}}\end{array}$ & $\begin{aligned} & 658.50 \\
\pm & 105.94^{\mathrm{ab}}\end{aligned}$ & $\begin{array}{c}599.50 \\
\pm 26.84^{\mathrm{bc}}\end{array}$ & 1991.5 & $\begin{array}{c}4.50 \\
\pm 0.29^{\mathrm{ab}}\end{array}$ & $\begin{array}{l}9.50 \\
\pm 0.29^{\mathrm{b}}\end{array}$ \\
\hline $\begin{array}{l}50 \% \text { Peat moss }+25 \% \\
\text { fronds }+25 \% \text { Vermi }\end{array}$ & $\begin{array}{c}28.0 \\
\pm 0.58^{\mathrm{c}}\end{array}$ & $\begin{array}{c}32.0 \\
\pm 0.58^{\mathrm{a}}\end{array}$ & $\begin{array}{l}37.00 \\
\pm 0.58^{\mathrm{de}}\end{array}$ & $\begin{array}{l}42.5 \\
\pm 0.58^{\mathrm{c}}\end{array}$ & $\begin{array}{l}880.5 \\
\pm 3.17^{\mathrm{a}}\end{array}$ & $\begin{array}{l}776.0 \\
\pm 7.50^{\mathrm{a}}\end{array}$ & $\begin{array}{l}810.00 \\
\pm 17.32^{\mathrm{a}}\end{array}$ & 2466.5 & $\begin{array}{l}5.00 \\
\pm 0.58^{\mathrm{a}}\end{array}$ & $\begin{array}{l}13.00 \\
\pm 0.00^{\mathrm{a}}\end{array}$ \\
\hline F. value & 1.885 & 2.100 & 17.200 & 32.500 & 10.960 & 6.476 & 16.219 & - & 4.591 & 424.500 \\
\hline Sig. & .131 & .097 & .000 & .000 & .000 & .001 & .000 & - & .004 & .000 \\
\hline
\end{tabular}

-Means with different letters in the same columns are significantly different $(\mathrm{P}<0.05)$ by Duncan multiple test. 
Table 2: Effect of casing materials on growth period, weight and shelf life of A.bisporus (S-2) strain.

\begin{tabular}{|c|c|c|c|c|c|c|c|c|c|c|}
\hline \multirow{2}{*}{ Casing media } & \multicolumn{4}{|c|}{$\begin{array}{l}\text { Growth period in days after spawning } \\
\qquad(\text { Mean } \pm \text { SE) }\end{array}$} & \multicolumn{3}{|c|}{$\begin{array}{l}\text { Weight (g) } \\
(\text { Mean } \pm \text { SE) }\end{array}$} & \multirow{2}{*}{$\begin{array}{c}\text { Total } \\
\text { Weight }(g)\end{array}$} & \multicolumn{2}{|c|}{$\begin{array}{l}\text { Shelf life (days) } \\
\quad(\text { Mean } \pm \text { SE) }\end{array}$} \\
\hline & $\begin{array}{l}\text { Pinhead } \\
\text { initiate }\end{array}$ & $\begin{array}{c}1^{\text {st }} \\
\text { Harv. }\end{array}$ & $\begin{array}{c}2^{\text {nd }} \\
\text { Harv. }\end{array}$ & $\begin{array}{c}3^{\text {rd }} \\
\text { Harv. }\end{array}$ & $\begin{array}{c}1^{\text {st }} \\
\text { Harv. }\end{array}$ & $\begin{array}{c}2^{\text {nd }} \\
\text { Harv. }\end{array}$ & $\begin{array}{c}3^{\text {rd }} \\
\text { Harv. }\end{array}$ & & $\begin{array}{l}\text { Fresh } \\
\text { air }\end{array}$ & Refrig. \\
\hline $\begin{array}{l}100 \% \text { Peat moss } \\
\text { (Control) }\end{array}$ & $\begin{array}{c}30.00 \\
\pm 0.29^{\mathrm{cd}}\end{array}$ & $\begin{array}{l}27.00 \\
\pm 2.31^{\mathrm{b}}\end{array}$ & $\begin{array}{c}37.00 \\
\pm 0.00^{\mathrm{bc}}\end{array}$ & $\begin{array}{c}43.50 \\
\pm 0.29^{\mathrm{ab}}\end{array}$ & $\begin{array}{l}334.00 \\
\pm 25.40^{\mathrm{f}}\end{array}$ & $\begin{array}{l}607.50 \\
\pm 21.65^{\mathrm{b}}\end{array}$ & $\begin{array}{c}565.50 \\
\pm 40.12^{\mathrm{bc}}\end{array}$ & 1507.0 & $\begin{array}{c}2.50 \\
\pm 0.29^{\mathrm{b}}\end{array}$ & $\begin{array}{l}10.00 \\
\pm 0.58^{\mathrm{b}}\end{array}$ \\
\hline $\begin{array}{c}75 \% \text { Peat moss }+25 \% \\
\text { Spent mushroom } \\
\text { substrate }\end{array}$ & $\begin{array}{c}30.00 \\
\pm 0.58^{\mathrm{ab}}\end{array}$ & $\begin{array}{l}33.00 \\
\pm 0.58^{\mathrm{a}}\end{array}$ & $\begin{array}{l}38.00 \\
\pm 0.58^{\mathrm{ab}}\end{array}$ & $\begin{array}{l}44.00 \\
\pm 0.58^{\mathrm{ab}}\end{array}$ & $\begin{array}{l}561.00 \\
\pm 60.62^{\text {cd }}\end{array}$ & $\begin{array}{l}540.50 \\
\pm 46.47^{\mathrm{b}}\end{array}$ & $\begin{array}{l}427.50 \\
\pm 102.48^{c}\end{array}$ & 1529.0 & $\begin{array}{l}3.50 \\
\pm 0.29^{\mathrm{b}}\end{array}$ & $\begin{array}{l}9.500 \\
\pm 0.29^{\mathrm{bc}}\end{array}$ \\
\hline $\begin{array}{l}75 \% \text { Peat moss }+25 \% \\
\text { Vermi }\end{array}$ & $\begin{array}{l}31.00 \\
\pm 0.58^{\mathrm{a}}\end{array}$ & $\begin{array}{l}33.50 \\
\pm 0.29^{\mathrm{a}}\end{array}$ & $\begin{array}{l}38.50 \\
\pm 0.29^{\mathrm{a}}\end{array}$ & $\begin{array}{l}44.50 \\
\pm 0.29^{\mathrm{a}}\end{array}$ & $\begin{array}{l}481.50 \\
\pm 47.05^{\mathrm{de}}\end{array}$ & $\begin{array}{l}397.50 \\
\pm 1.44^{\text {cd }}\end{array}$ & $\begin{array}{l}423.00 \\
\pm 19.63^{\mathrm{c}}\end{array}$ & 1302.0 & $\begin{array}{c}5.00 \\
\pm 0.00^{\mathrm{a}}\end{array}$ & $\begin{array}{l}11.500 \\
\pm 0.29^{\mathrm{a}}\end{array}$ \\
\hline $\begin{array}{c}50 \% \text { Peat moss }+25 \% \\
\text { Spent mushroom }+ \\
25 \% \text { Vermi } \\
\end{array}$ & $\begin{array}{c}29.50 \\
\pm 0.29^{\mathrm{bc}}\end{array}$ & $\begin{array}{l}32.50 \\
\pm 0.29^{\mathrm{a}}\end{array}$ & $\begin{array}{c}36.00 \\
\pm 0.58^{\mathrm{cd}}\end{array}$ & $\begin{array}{l}42.50 \\
\pm 0.87^{\mathrm{bc}}\end{array}$ & $\begin{array}{l}383.00 \\
\pm 34.64^{\mathrm{ef}}\end{array}$ & $\begin{array}{c}481.50 \\
\pm 33.77^{\mathrm{bc}}\end{array}$ & $\begin{array}{l}629.50 \\
\pm 42.43^{\mathrm{b}}\end{array}$ & 1494.0 & $\begin{aligned} & 2.50 \\
\pm & 0.29^{\mathrm{b}}\end{aligned}$ & $\begin{array}{l}8.500 \\
\pm 0.29^{\text {cd }}\end{array}$ \\
\hline $\begin{array}{l}50 \% \text { Peat moss }+50 \% \\
\text { fronds }\end{array}$ & $\begin{array}{c}29.50 \\
\pm 0.29^{\mathrm{bc}}\end{array}$ & $\begin{array}{l}32.50 \\
\pm 0.29^{\mathrm{a}}\end{array}$ & $\begin{array}{c}37.00 \\
\pm 0.58^{\mathrm{bc}}\end{array}$ & $\begin{array}{l}43.00 \\
\pm 0.29^{\mathrm{a}}\end{array}$ & $\begin{array}{c}698.00 \\
\pm 23.09^{\mathrm{b}}\end{array}$ & $\begin{array}{c}489.50 \\
\pm 89.78^{\mathrm{bc}}\end{array}$ & $\begin{array}{r}619.00 \\
\pm 37.52^{\mathrm{b}}\end{array}$ & 1806.5 & $\begin{array}{l}2.50 \\
\pm 0.29^{\mathrm{b}}\end{array}$ & $\begin{array}{l}8.00 \\
\pm 0.58^{\mathrm{d}}\end{array}$ \\
\hline $\begin{array}{l}50 \% \text { Peat moss }+25 \% \\
\text { fronds }+25 \% \text { Vermi }\end{array}$ & $\begin{array}{l}29.00 \\
\pm 0.58^{\mathrm{b}}\end{array}$ & $\begin{array}{l}31.00 \\
\pm 0.58^{\mathrm{a}}\end{array}$ & $\begin{array}{l}34.00 \\
\pm 0.58^{\mathrm{e}}\end{array}$ & $\begin{array}{l}39.00 \\
\pm 0.58^{\mathrm{d}}\end{array}$ & $\begin{array}{l}900.50 \\
\pm 33.19^{\mathrm{a}}\end{array}$ & $\begin{array}{l}823.00 \\
\pm 17.32^{\mathrm{a}}\end{array}$ & $\begin{array}{l}819.00 \\
\pm 17.89^{\mathrm{a}}\end{array}$ & 2542.5 & $\begin{array}{l}4.50 \\
\pm 0.29^{\mathrm{a}}\end{array}$ & $\begin{array}{r}9.00 \\
\pm 0.00^{\mathrm{bcc}}\end{array}$ \\
\hline F. value & 5.000 & 5.421 & 11.056 & 15.952 & 23.825 & 16.696 & 8.311 & - & 9.889 & 8.800 \\
\hline Sig. & .003 & .002 & .000 & .000 & .000 & .000 & .000 & - & .000 & .000 \\
\hline
\end{tabular}

-Means with different letters in the same columns are significantly different $(\mathrm{P}<0.05)$ by Duncan multiple test. 
Table 3: Effect of casing materials on total fruits number of two A. bisporus tested strains.

\begin{tabular}{|c|c|c|c|c|c|c|c|c|}
\hline \multirow{3}{*}{ Casing media } & \multicolumn{8}{|c|}{ Number of fruit $($ Mean \pm SE) } \\
\hline & \multicolumn{4}{|c|}{ Strain-1 } & \multicolumn{4}{|c|}{ Strain-2 } \\
\hline & $1^{\text {st }}$ Flush & $2^{\text {nd }}$ flush & $3^{\text {rd }}$ flush & $\begin{array}{l}\text { Total } \\
\text { No. }\end{array}$ & $1^{\text {st }}$ flush & $2^{\text {nd }}$ flush & $3^{\text {rd }}$ flush & Total No. \\
\hline $100 \%$ Peat moss (Control) & $\begin{array}{l}25.00 \\
\pm 2.31^{\mathrm{bc}}\end{array}$ & $\begin{array}{l}27.50 \\
\pm 1.44^{\mathrm{ab}}\end{array}$ & $\begin{aligned} & 27.50 \\
\pm & 2.02^{\mathrm{abc}}\end{aligned}$ & 81.5 & $\begin{array}{l}28.50 \\
\pm 0.87^{\mathrm{b}}\end{array}$ & $\begin{array}{l}28.50 \\
\pm 0.87^{\mathrm{ab}}\end{array}$ & $\begin{array}{l}24.50 \\
\pm 2.02^{\mathrm{bc}}\end{array}$ & 80.0 \\
\hline $75 \%$ Peat moss + 25\% Spent mushroom & $\begin{array}{l}25.50 \\
\pm 2.56^{\mathrm{bc}}\end{array}$ & $\begin{array}{l}23.50 \\
\pm 0.87^{\mathrm{b}}\end{array}$ & $\begin{array}{c}28.00 \\
\pm 2.31^{\mathrm{ab}}\end{array}$ & 77.0 & $\begin{array}{l}26.00 \\
\pm 0.00^{\mathrm{c}}\end{array}$ & $\begin{array}{l}24.50 \\
\pm 0.87^{\text {bcd }}\end{array}$ & $\begin{array}{c}29.00 \\
\pm 0.58^{\mathrm{ab}}\end{array}$ & 79.5 \\
\hline $75 \%$ Peat moss $+25 \%$ Vermi & $\begin{array}{l}22.50 \\
\pm 0.87^{\mathrm{a}}\end{array}$ & $\begin{array}{l}23.50 \\
\pm 2.02^{\mathrm{b}}\end{array}$ & $\begin{array}{l}24.00 \\
\pm 0.58^{\mathrm{bc}}\end{array}$ & 70.0 & $\begin{array}{l}29.00 \\
\pm 0.58^{b}\end{array}$ & $\begin{aligned} & 26.00 \\
\pm & 1.73^{\mathrm{abc}}\end{aligned}$ & $\begin{aligned} & 28.50 \\
\pm & 1.44^{\mathrm{abc}}\end{aligned}$ & 83.5 \\
\hline $\begin{array}{l}50 \% \text { Peat moss }+25 \% \text { Spent mushroom }+25 \% \\
\text { Vermi }\end{array}$ & $\begin{array}{c}24.00 \\
\pm 0.58^{\mathrm{bc}}\end{array}$ & $\begin{array}{c}27.00 \\
\pm 0.58^{\mathrm{ab}}\end{array}$ & $\begin{array}{c}23.50 \\
\pm 0.29^{\mathrm{c}}\end{array}$ & 74.5 & $\begin{array}{c}25.00 \\
\pm 1.15^{\mathrm{c}}\end{array}$ & $\begin{array}{c}21.50 \\
\pm 0.87^{\mathrm{d}}\end{array}$ & $\begin{array}{c}27.00 \\
\pm 0.58^{\mathrm{abc}}\end{array}$ & 73.5 \\
\hline $50 \%$ Peat moss $+50 \%$ fronds & $\begin{array}{c}27.00 \\
\pm 2.89^{\mathrm{bc}}\end{array}$ & $\begin{array}{c}25.50 \\
\pm 0.29^{\mathrm{b}}\end{array}$ & $\begin{array}{c}27.50 \\
\pm 0.29^{\mathrm{abc}}\end{array}$ & 80.0 & $\begin{array}{c}33.00 \\
\pm 0.58^{\mathrm{a}}\end{array}$ & $\begin{array}{c}23.50 \\
\pm 2.59^{\text {cd }}\end{array}$ & $\begin{array}{c}29.50 \\
\pm 2.02^{\mathrm{a}}\end{array}$ & 86.0 \\
\hline $50 \%$ Peat moss $+25 \%$ fronds $+25 \%$ Vermi & $\begin{array}{l}33.50 \\
\pm 0.29^{\mathrm{a}}\end{array}$ & $\begin{array}{c}30.50 \\
\pm 0.29^{\mathrm{a}}\end{array}$ & $\begin{array}{l}29.00 \\
\pm 0.00^{\mathrm{a}}\end{array}$ & 93.0 & $\begin{array}{c}29.50 \\
\pm 0.87^{\mathrm{b}}\end{array}$ & $\begin{array}{l}29.50 \\
\pm 0.29^{\mathrm{a}}\end{array}$ & $\begin{array}{c}29.50 \\
\pm 0.87^{\mathrm{a}}\end{array}$ & 88.5 \\
\hline F. value & 4.116 & 4.504 & 2.516 & - & 30.884 & 5.500 & 2.602 & - \\
\hline Sig. & 0.007 & 0.004 & 0.054 & - & 0.000 & .001 & .048 & - \\
\hline
\end{tabular}

Means with different letters in the same columns are significantly different $(\mathrm{P}<0.05)$ by Duncan multiple test 
Table 4: Effect of casing materials on fruit weight of two A.bisporus tested strains.

\begin{tabular}{|c|c|c|c|c|c|c|c|c|}
\hline \multirow{3}{*}{ Casing media } & \multicolumn{8}{|c|}{ Fruit weight (g) of Agaricus bisporus (Mean \pm SE) } \\
\hline & \multirow[b]{2}{*}{$1^{\text {st }}$ flush } & \multicolumn{2}{|l|}{ Strain-1 } & \multirow[b]{2}{*}{ Total wt. } & \multicolumn{4}{|c|}{ Strain-2 } \\
\hline & & $2^{\text {nd }}$ flush & $3^{\text {rd }}$ flush & & $1^{\text {st }}$ flush & $2^{\text {nd }}$ flush & $3^{\text {rd }}$ flush & Total wt. \\
\hline $100 \%$ Peat moss (Control) & $\begin{array}{l}19.20 \\
\pm 0.94^{\mathrm{c}}\end{array}$ & $\begin{array}{l}21.15 \\
\pm 1.09^{\mathrm{b}}\end{array}$ & $\begin{array}{l}22.10 \\
\pm 0.97^{\mathrm{ab}}\end{array}$ & 62.8 & $\begin{array}{l}21.70 \\
\pm 0.500^{\mathrm{b}}\end{array}$ & $\begin{array}{l}19.80 \\
\pm 0.74^{\mathrm{a}}\end{array}$ & $\begin{array}{l}21.30 \\
\pm 0.50^{\mathrm{bc}}\end{array}$ & 62.8 \\
\hline $75 \%$ Peat moss $+25 \%$ Spent mushroom & $\begin{array}{l}21.55 \\
\pm 0.38^{\mathrm{ab}}\end{array}$ & $\begin{array}{l}21.50 \\
\pm 0.45^{\mathrm{b}}\end{array}$ & $\begin{array}{l}18.90 \\
\pm 0.93^{\mathrm{c}}\end{array}$ & 61.9 & $\begin{array}{l}18.20 \\
\pm 0.63^{\text {de }}\end{array}$ & $\begin{array}{l}20.10 \\
\pm 0.75^{\mathrm{a}}\end{array}$ & $\begin{array}{l}20.55 \\
\pm 0.42^{\mathrm{bc}}\end{array}$ & 58.8 \\
\hline $75 \%$ Peat moss $+25 \%$ Vermi & $\begin{array}{l}20.80 \\
\pm 0.70^{\mathrm{abc}}\end{array}$ & $\begin{array}{c}18.40 \\
\pm 0.73^{\mathrm{c}}\end{array}$ & $\begin{array}{c}18.80 \\
\pm 0.36^{\mathrm{c}}\end{array}$ & 58.0 & $\begin{array}{c}20.15 \\
\pm 1.05^{\text {bcd }}\end{array}$ & $\begin{array}{c}19.20 \\
\pm 0.60^{\mathrm{a}}\end{array}$ & $\begin{array}{c}19.95 \\
\pm 0.78^{\mathrm{c}}\end{array}$ & 59.3 \\
\hline $\begin{array}{l}50 \% \text { Peat moss }+25 \% \text { Spent mushroom }+ \\
25 \% \text { Vermi- }\end{array}$ & $\begin{array}{c}19.40 \\
\pm 0.87^{\mathrm{bc}}\end{array}$ & $\begin{array}{l}18.25 \\
\pm 0.71^{\mathrm{c}}\end{array}$ & $\begin{array}{c}19.85 \\
\pm 0.77^{\mathrm{bc}}\end{array}$ & 57.5 & $\begin{array}{c}19.60 \\
\pm 0.44^{\text {cde }}\end{array}$ & $\begin{array}{c}12.73 \\
\pm 4.15^{\mathrm{b}}\end{array}$ & $\begin{array}{c}20.65 \\
\pm 0.64^{\mathrm{bc}}\end{array}$ & 52.9 \\
\hline $50 \%$ Peat moss $+50 \%$ fronds & $\begin{array}{c}20.15 \\
\pm 0.58^{\mathrm{bc}} \\
\end{array}$ & $\begin{array}{c}19.95 \\
\pm 1.07^{\mathrm{bc}} \\
\end{array}$ & $\begin{array}{c}19.10 \\
\pm 0.52^{\mathrm{c}}\end{array}$ & 59.2 & $\begin{array}{c}20.70 \\
\pm 0.78^{\mathrm{bc}}\end{array}$ & $\begin{array}{c}19.85 \\
\pm 0.63^{\mathrm{a}}\end{array}$ & $\begin{array}{c}20.45 \\
\pm 0.78^{\mathrm{bc}}\end{array}$ & 61.0 \\
\hline $50 \%$ Peat moss $+25 \%$ fronds $+25 \%$ Vermi & $\begin{array}{c}22.70 \\
\pm 0.25^{\mathrm{a}}\end{array}$ & $\begin{array}{l}24.30 \\
\pm 0.45^{\mathrm{a}}\end{array}$ & $\begin{array}{c}23.30 \\
\pm 0.46^{\mathrm{a}}\end{array}$ & 70.3 & $\begin{array}{c}24.00 \\
\pm 0.18^{\mathrm{a}}\end{array}$ & $\begin{array}{l}24.00 \\
\pm 0.24^{\mathrm{a}}\end{array}$ & $\begin{array}{l}24.45 \\
\pm 0.25^{\mathrm{a}}\end{array}$ & 72.4 \\
\hline F. value & 3.395 & 6.292 & 4.184 & - & 10.555 & 3.823 & 7.110 & - \\
\hline Sig. & 0.017 & 0.001 & 0.006 & & 0.000 & 0.010 & 0.000 & \\
\hline
\end{tabular}

Means with different letters in the same columns are significantly different $(\mathrm{P}<0.05)$ by Duncan multiple test. 
mushroom fruits of strain (S-2) was 88.5 in the treatment covered with peat moss + palm fronds + vermicompost while the control casing of strain (S-2) recorded 80 fruits. Both strains showed significant differences between treatments in the first and second flush while the differences between treatments at the third flush were insignificant.

\subsection{Effect of casing materials on fruit weight of two A.bisporus tested strains.}

Data presented in Table (4) showed that the maximum weight of mushroom fruits of strain (S-1) and (S-2) were 70.3 and $72.49 \mathrm{~g}$, respectively, obtained in the treatment covered with peat moss + palm fronds + vermicompost, while the minimum weight was 57.5 and $52.9 \mathrm{~g}$ obtained in the treatment covered with peat moss + spent mushroom + vermicompost. When the casing of both strains was peat moss only, the average total weight gave $62.8 \mathrm{~g}$. In general, weight of mushroom fruits in treatments covered with peat moss + palm fronds + vermicompost for both strains was significantly higher compared to other casing layers.

Kumar et al., (2020) studied Physical parameters of casing materials on yield parameter of white button mushroom, they found that, fresh and dry matter were increased on fruiting body of mushroom with maximum in FYM + Soil + Sand (2:1:1), representing 1192.41 and $173.42 \mathrm{~g}$, against $763.89 \mathrm{~g}$ and $82.8 \mathrm{~g}$ in case of control.

\subsection{Effect of casing materials on stipe length and stipe breadth}

Fruit stipe length of both strains was generally longer for the first harvest compared to those of the second and third harvests. However, it was noticed that breadth of stipe was decreased with increasing length (Tables 5 and 6). Fruit stipe length of (S-1) and (S-2) strains in treatment of peat moss + palm fronds + vermicompost was $(4.61,2.35$ and $2.23 \mathrm{~cm})$ and $(3.47,3.26$ and $3.10 \mathrm{~cm})$ in $1^{\text {st }}, 2^{\text {nd }}$ and $3^{\text {rd }}$ flushes, respectively. Also fruit stipe breadth of the (S-1) and (S-2) in the same treatment was (1.46. 1.77 and $1.78 \mathrm{~cm})$ and $(1.56,1.6$ and $2.02 \mathrm{~cm})$, respectively. In general al, both two strains showed insignificant differences compared with other treatments in the first, second and third flushes for fruit stipe length and breadth of stipe. However, in terms of fruit stipe length, S-1strran showed significant differences $(\mathrm{p}<0.05)$ at the $2^{\text {nd }}$ and $3^{\text {rd }}$ flushes. In a similar experiment, Kumar et al., (2020) found that, the maximum length of white button mushroom stalk $(\mathrm{cm})$ was $3.04 \mathrm{~cm}$ in FYM + Soil + Sand $(2: 1: 1)$, followed by $2.97 \mathrm{~cm}$ in FYM + Soil + burnt rice husk (Ash) (2:1:1) and $2.94 \mathrm{~cm}$ in FYM + Sand + Ash (3:1:1/2). They found also the maximum diameter and thickness of pileus $(\mathrm{cm})$ in FYM + Soil + Sand (2:1:1), representing $5.49 \mathrm{~cm}$ and $1.89 \mathrm{~cm}$. against 4.01 and $1.14 \mathrm{~cm}$ in case of control.

\subsection{Effect of casing materials on pileus diameter and thickness}

Fruit pileus diameter and thickness of both strains were generally larger for the first harvest compared to those of the second and third harvests (Tables 7 and 8 ). In the $1^{\text {st }}$ harvest, fruit pileus diameter and thickness of peat moss + palm fronds + vermicompost treatment exhibited the highest values (3.78 and $1.99 \mathrm{~cm}$ for S-1 strain and 3.90 and $1.92 \mathrm{~cm}$ for S-2 strain, respectively) followed by those of peat moss + palm fronds treatment. Both two strains showed insignificant differences between treatments in the $1^{\text {st }}, 2^{\text {nd }}$ and $3^{\text {rd }}$ flushes for fruit pileus diameter and thickness except treatments at first flush for fruit pileus diameter of strain (S-1) and third flush for fruit pileus diameter of strain (S-2), the differences were significant $(P<0.05)$.

\subsection{Effect of casing materials on phenolics, flavonoids and antioxidants}

Phenolic compounds belong to bioactive compounds, although they are non-essential dietary components. Their biological function is related to free radical scavenging activity, metal chelation ability and inhibition of lipid oxidation (Cheung et al., 2003). There are many diseases such as heart disease, cancer, arthritis, and the aging process itself, in which free radicals are implicated. To combat these free radicals the body needs antioxidants (Harman, 1997) and in this respect, flavonoids and other phenols have shown to possess an important antioxidant activity towards these radicals, which is principally based on the redox properties of their phenolic hydroxyl groups and the structural relationships between different parts of their chemical structure (Bors and Saran, 1987). Antioxidant compounds can increase shelf life by retarding the process of lipid peroxidation, which is also one of the major reasons for deterioration of food products during processing and storage (Halliwell, 1997). Thus a need for identifying sources of antioxidants has been created, and the search for natural 
Table 5: Impact of casing materials of $A$. bisporus (S-1) strain on stipe length and stipe breadth.

\begin{tabular}{|c|c|c|c|c|c|c|}
\hline \multirow{3}{*}{ Casing media } & \multicolumn{6}{|c|}{ Fruit stipe (Mean \pm SE) } \\
\hline & \multicolumn{3}{|c|}{ Stipe length (cm) } & \multicolumn{3}{|c|}{ Stipe breadth (cm) } \\
\hline & $1^{\text {st }}$ flush & $2^{\text {nd }}$ flush & $3^{\text {rd }}$ flush & $1^{\text {st }}$ flush & $2^{\text {nd }}$ flush & $3^{\text {rd }}$ Flush \\
\hline $100 \%$ Peat moss(Control) & $2.77 \pm 0.18^{\mathrm{b}}$ & $2.64 \pm 0.25^{\mathrm{b}}$ & $2.25 \pm 0.47^{\mathrm{ab}}$ & $1.77 \pm 0.14^{\mathrm{ab}}$ & $1.82 \pm 0.11^{\mathrm{a}}$ & $1.84 \pm 0.09^{\mathrm{a}}$ \\
\hline $75 \%$ Peat moss $+25 \%$ Spent mushroom & $2.91 \pm 0.15^{\mathrm{ab}}$ & $2.44 \pm 0.12^{b}$ & $2.34 \pm 0.18^{\mathrm{b}}$ & $1.80 \pm 0.11^{\mathrm{a}}$ & $1.82 \pm 0.12^{\mathrm{a}}$ & $1.82 \pm 0.11^{\mathrm{a}}$ \\
\hline $75 \%$ Peat moss $+25 \%$ Vermi & $3.25 \pm 0.31^{\mathrm{a}}$ & $3.21 \pm 0.17^{\mathrm{a}}$ & $3.20 \pm 0.16^{\mathrm{a}}$ & $1.60 \pm 0.09^{\mathrm{ab}}$ & $1.82 \pm 0.08^{\mathrm{a}}$ & $1.88 \pm 0.11^{\mathrm{a}}$ \\
\hline $50 \%$ Peat moss $+25 \%$ Spent mushroom $+25 \%$ Vermi & $3.40 \pm 0.21^{\mathrm{a}}$ & $3.28 \pm 0.03^{\mathrm{a}}$ & $3.23 \pm 0.17^{\mathrm{ab}}$ & $1.69 \pm 0.02^{\mathrm{ab}}$ & $1.74 \pm 0.02^{\mathrm{a}}$ & $1.79 \pm 0.02^{\mathrm{a}}$ \\
\hline $50 \%$ Peat moss $+50 \%$ fronds & $3.42 \pm 0.13^{\mathrm{a}}$ & $3.41 \pm 0.14^{\mathrm{a}}$ & $3.23 \pm 0.16^{\mathrm{a}}$ & $1.58 \pm 0.06^{\mathrm{ab}}$ & $1.63 \pm 0.05^{\mathrm{a}}$ & $1.68 \pm 0.09^{\mathrm{a}}$ \\
\hline $50 \%$ Peat moss $+25 \%$ fronds $+25 \%$ Vermi & $4.61 \pm 0.17^{\mathrm{a}}$ & $2.35 \pm 0.18^{\mathrm{b}}$ & $2.23 \pm 0.23^{\mathrm{b}}$ & $1.46 \pm 0.05^{\mathrm{b}}$ & $1.77 \pm 0.14^{\mathrm{a}}$ & $1.78 \pm 0.14^{\mathrm{a}}$ \\
\hline F. value & 2.198 & 7.308 & 3.263 & 1.948 & 0.559 & 1.381 \\
\hline Sig. & 0.084 & 0.000 & 0.020 & 0.120 & 0.758 & 0.268 \\
\hline
\end{tabular}

-Means with different letters in the same columns are significantly different $(\mathrm{P}<0.05)$ by Duncan multiple test.

Table 6: Impact of casing materials of A. bisporus (S-2) strain on stipe length and stipe breadth.

\begin{tabular}{|c|c|c|c|c|c|c|}
\hline \multirow{3}{*}{ Casing media } & \multicolumn{6}{|c|}{ Fruit stipe (Mean \pm SE) } \\
\hline & \multicolumn{3}{|c|}{ Stipe length (cm) } & \multicolumn{3}{|c|}{ Stipe breadth $(\mathrm{cm})$} \\
\hline & $1^{\text {st }}$ flush & $2^{\text {nd }}$ flush & $3^{\text {rd }}$ flush & $1^{\text {st }}$ flush & $2^{\text {nd }}$ flush & $3^{\text {rd }}$ flush \\
\hline $100 \%$ Peat moss (Control) & $3.08 \pm 0.47^{\mathrm{a}}$ & $2.78 \pm 0.20^{\mathrm{ab}}$ & $2.73 \pm 0.51^{\mathrm{a}}$ & $1.82 \pm 0.11^{\mathrm{a}}$ & $1.82 \pm 0.11^{\mathrm{a}}$ & $1.87 \pm 0.13$ \\
\hline $75 \%$ Peat moss $+25 \%$ Spent mushroom & $3.23 \pm 0.04^{\mathrm{a}}$ & $3.22 \pm 0.26^{\mathrm{b}}$ & $2.69 \pm 0.06^{\mathrm{a}}$ & $1.54 \pm 0.05^{\mathrm{b}}$ & $1.61 \pm 0.08^{\mathrm{a}}$ & $1.88 \pm 0.03$ \\
\hline $75 \%$ Peat moss $+25 \%$ Vermi & $3.33 \pm 0.17^{\mathrm{a}}$ & $3.23 \pm 0.11^{\mathrm{ab}}$ & $3.16 \pm 0.13^{\mathrm{a}}$ & $1.64 \pm 0.05^{\mathrm{ab}}$ & $1.70 \pm 0.04^{\mathrm{a}}$ & $1.83 \pm 0.04$ \\
\hline $\begin{array}{l}50 \% \text { Peat moss }+25 \% \text { Spent mushroom }+25 \% \\
\text { Vermi }\end{array}$ & $3.39 \pm 0.07^{\mathrm{a}}$ & $3.11 \pm 0.17^{\mathrm{a}}$ & $3.02 \pm 0.31^{\mathrm{a}}$ & $1.67 \pm 0.01^{\mathrm{ab}}$ & $1.70 \pm 0.02^{\mathrm{ab}}$ & $1.75 \pm 0.07$ \\
\hline $50 \%$ Peat moss $+50 \%$ fronds & $3.43 \pm 0.16^{\mathrm{a}}$ & $3.26 \pm 0.08^{\mathrm{ab}}$ & $2.80 \pm 1.42^{\mathrm{a}}$ & $1.57 \pm 0.06^{\mathrm{ab}}$ & $1.58 \pm 0.06^{\mathrm{b}}$ & $1.89 \pm 0.49^{2}$ \\
\hline $50 \%$ Peat moss $+25 \%$ fronds $+25 \%$ Vermi & $3.47 \pm 0.19^{\mathrm{a}}$ & $3.26 \pm 0.48^{\mathrm{ab}}$ & $3.10 \pm 0.12^{\mathrm{a}}$ & $1.56 \pm 0.10^{\mathrm{b}}$ & $1.60 \pm 0.07^{\mathrm{a}}$ & $2.02 \pm 0.07$ \\
\hline F. value & 0.624 & 1.486 & 1.029 & 2.023 & 2.020 & 0.595 \\
\hline Sig. & 0.709 & 0.231 & 0.434 & 0.108 & 0.108 & 0.731 \\
\hline
\end{tabular}

-Means with different letters in the same columns are significantly different $(\mathrm{P}<0.05)$ by Duncan multiple test. 
Table 7: Impact of casing materials of A. bisporus (S-1) strain on pileus diameter and pileus thickness

\begin{tabular}{|c|c|c|c|c|c|c|}
\hline \multirow{3}{*}{ Casing media } & \multicolumn{6}{|c|}{ Fruit pileus (Mean \pm SE) } \\
\hline & \multicolumn{3}{|c|}{ Pileus diameter (cm) } & \multicolumn{3}{|c|}{ Pileus thickness (cm) } \\
\hline & $1^{\text {st flush }}$ & $2^{\text {nd }}$ flush & $3^{\text {rd }}$ flush & $1^{\text {st flush }}$ & $2^{\text {nd }}$ flush & $3^{\text {rd }}$ flush \\
\hline $100 \%$ Peat moss (Control) & $2.99 \pm 0.08^{\mathrm{bc}}$ & $2.87 \pm 0.16^{\mathrm{b}}$ & $2.62 \pm 0.27^{\mathrm{a}}$ & $1.97 \pm 0.11^{\mathrm{a}}$ & $1.92 \pm 0.16^{\mathrm{a}}$ & $1.84 \pm 0.12^{\mathrm{a}}$ \\
\hline $75 \%$ Peat moss $+25 \%$ Spent mushroom & $2.76 \pm 0.19^{c}$ & $2.73 \pm 0.21^{\mathrm{ab}}$ & $2.72 \pm 0.19^{\mathrm{a}}$ & $1.99 \pm 0.07^{\mathrm{a}}$ & $1.88 \pm 0.09^{\mathrm{a}}$ & $1.75 \pm 0.14^{\mathrm{a}}$ \\
\hline $75 \%$ Peat moss $+25 \%$ Vermi & $3.37 \pm 0.16^{\mathrm{bc}}$ & $3.12 \pm 0.35^{\mathrm{ab}}$ & $2.99 \pm 0.10^{\mathrm{a}}$ & $1.97 \pm 0.09^{\mathrm{a}}$ & $1.91 \pm 0.11^{\mathrm{a}}$ & $1.74 \pm 0.12^{\mathrm{a}}$ \\
\hline $50 \%$ Peat moss $+25 \%$ Spent mushroom $+25 \%$ Vermi & $3.37 \pm 0.14^{\mathrm{ab}}$ & $3.26 \pm 0.11^{\mathrm{a}}$ & $2.69 \pm 0.22^{\mathrm{a}}$ & $1.76 \pm 0.06^{\mathrm{a}}$ & $1.76 \pm 0.06^{\mathrm{a}}$ & $1.75 \pm 0.08^{\mathrm{ab}}$ \\
\hline $50 \%$ Peat moss $+50 \%$ fronds & $3.57 \pm 0.08^{\mathrm{ab}}$ & $3.27 \pm 0.09^{\mathrm{a}}$ & $3.17 \pm 0.09^{\mathrm{a}}$ & $1.72 \pm 0.05^{\mathrm{a}}$ & $1.72 \pm 0.03^{\mathrm{a}}$ & $1.64 \pm 0.06^{\mathrm{ab}}$ \\
\hline $50 \%$ Peat moss $+25 \%$ fronds $+25 \%$ Vermi & $3.78 \pm 0.03^{\mathrm{ab}}$ & $2.98 \pm 0.15^{\mathrm{b}}$ & $2.64 \pm 0.33^{\mathrm{a}}$ & $1.99 \pm 0.15^{\mathrm{a}}$ & $1.99 \pm 0.09^{\mathrm{a}}$ & $1.76 \pm 0.09^{\mathrm{a}}$ \\
\hline F. value & 3.575 & 2.139 & 1.278 & .258 & 1.374 & 2.665 \\
\hline Sig. & 0.013 & 0.091 & 0.310 & 0.951 & 0.270 & .0440 \\
\hline
\end{tabular}

-Means with different letters in the same columns are significantly different $(\mathrm{P}<0.05)$ by Duncan multiple test.

Table 8: Effect of casing materials of A. bisporus (S-2) strain on pileus diameter and pileus thickness.

\begin{tabular}{|c|c|c|c|c|c|c|}
\hline \multirow{3}{*}{ Casing media } & \multicolumn{6}{|c|}{ Fruit pileus (Mean \pm SE) } \\
\hline & \multicolumn{3}{|c|}{ Pileus diameter $(\mathrm{cm})$} & \multicolumn{3}{|c|}{ Pileus thickness (cm) } \\
\hline & $1^{\text {st }}$ flush & $2^{\text {nd }}$ flush & $3^{\text {rd }}$ flush & $1^{\text {st }}$ flush & $2^{\text {nd }}$ flush & $3^{\text {rd }}$ flush \\
\hline $100 \%$ Peat moss (Control) & $2.95 \pm 0.31^{\mathrm{a}}$ & $2.87 \pm 0.28^{\mathrm{a}}$ & $2.77 \pm 0.22^{\mathrm{c}}$ & $1.90 \pm 0.13^{\mathrm{a}}$ & $1.90 \pm 0.16^{\mathrm{a}}$ & $1.87 \pm 0.14^{\mathrm{a}}$ \\
\hline $75 \%$ Peat moss $+25 \%$ Spent mushroom & $3.44 \pm 0.28^{\mathrm{a}}$ & $2.86 \pm 0.25^{\mathrm{a}}$ & $2.82 \pm 0.10^{\mathrm{a}}$ & $1.72 \pm 0.09^{\mathrm{a}}$ & $1.70 \pm 0.09^{\mathrm{a}}$ & $1.65 \pm 0.03^{\mathrm{a}}$ \\
\hline $75 \%$ Peat moss $+25 \%$ Vermi & $3.43 \pm 0.16^{\mathrm{a}}$ & $3.12 \pm 0.13^{\mathrm{a}}$ & $3.08 \pm 0.06^{\mathrm{abc}}$ & $1.75 \pm 0.07^{\mathrm{a}}$ & $1.73 \pm 0.06^{\mathrm{a}}$ & $1.67 \pm 0.07^{\mathrm{a}}$ \\
\hline $50 \%$ Peat moss $+25 \%$ Spent mushroom $+25 \%$ Vermi & $3.42 \pm 0.14^{\mathrm{a}}$ & $3.25 \pm 0.16^{\mathrm{a}}$ & $3.19 \pm 0.12^{\mathrm{a}}$ & $1.77 \pm 0.07^{\mathrm{a}}$ & $1.71 \pm 0.06^{\mathrm{a}}$ & $1.63 \pm 0.05^{\mathrm{a}}$ \\
\hline $50 \%$ Peat moss $+50 \%$ fronds & $3.50 \pm 0.16^{\mathrm{a}}$ & $3.40 \pm 0.19^{\mathrm{a}}$ & $3.18 \pm 0.18^{\mathrm{ab}}$ & $1.77 \pm 0.06^{\mathrm{a}}$ & $1.66 \pm 0.05^{\mathrm{a}}$ & $1.65 \pm 0.06^{\mathrm{a}}$ \\
\hline $50 \%$ Peat moss $+25 \%$ fronds $+25 \%$ Vermi & $3.90 \pm 1.53^{\mathrm{a}}$ & $3.41 \pm 0.23^{\mathrm{a}}$ & $2.79 \pm 0.09^{\mathrm{a}}$ & $1.92 \pm 0.16^{\mathrm{a}}$ & $1.84 \pm 0.12^{\mathrm{a}}$ & $1.68 \pm 0.09^{\mathrm{a}}$ \\
\hline F. value & 0.568 & 1.842 & 3.031 & 1.299 & 1.263 & 0.663 \\
\hline Sig. & 0.751 & 0.139 & 0.027 & 0.300 & 0.316 & 0.680 \\
\hline
\end{tabular}

-Means with different letters in the same columns are significantly different $(\mathrm{P}<0.05)$ by Duncan multiple test. 
antioxidants, especially of natural origin, has notably increased in recent years (Skerget et al., 2005). Mushrooms have become attractive as a functional food and as source for the development of drugs and nutraceutics, namely for antioxidant compounds (Lo and Cheung, 2005).

In our results, phenolic compounds contents ranged from 237.1 to $218.47 \mathrm{mg}$ dry weigh and the maximum content of total phenolics in (S-1) strain was observed in treatment of $100 \%$ peat moss $(237.1$ $\mathrm{mg} / \mathrm{g})$, while the minimum was recorded with peat moss + spent mushroom $(218.47 \mathrm{mg} / \mathrm{g})$ treatment (Table 9). The differences between all casing media of A. bisporus (S-1) for total phenolics content were insignificant. Flavonoids content ranged from 57.21 to $50.39 \mathrm{mg} / \mathrm{g}$ dry weigh and showed insignificant values between casing layer treatments except treatment peat moss + frond which was significantly higher compared to other treatments. Flavonoids content ranged between 50.39 and 57.21 $\mathrm{mg} / \mathrm{g}$ for peat moss + spent mushroom + vermicompost and peat moss with frond, respectively. The highest content of antioxidant activity was observed in control treatment $(72.88 \%)$ while the lowest activity $(50.20 \%)$ was observed in peat moss + frond + vermicompost treatment. The differences between all treatments and control were significant. On the other hand, in case of (S-2) strain, the highest content of phenolics ( $252.93 \mathrm{mg} / \mathrm{g}$ dry weigh) was recorded by the treatment That contained Peat moss + vermicompost whereas peat moss + spent mushroom recorded the lowest $(208.70 \mathrm{mg} / \mathrm{g}$ dry weigh). The highest flavonoids content $(54.79 \mathrm{mg} /$ dry weigh) and antioxidant $(69.47 \%)$ values in strain (S-2) were observed in the control treatment where the casing layer contained peat moss only (Table 10). Also the same treatment Peat moss + spent mushroom recorded the lowest values of both contents $(46.81 \mathrm{mg} / 1$ dry weigh and $42.80 \%$, respectively). In general, total phenolic compounds, flavonoids content and antioxidant activity showed high significance between treatments at $(P<0.05)$. In another study by Mami et al., (2013), they found that total phenol and antioxidant were increased in the compost of $A$. bisporus when supplemented with ground corn and soybean seed as a casing layer.

Table 9: Effect of casing materials of A. bisporus (S-1) strain for phenolics, flavonoids and antioxidant.

\begin{tabular}{|c|c|c|c|}
\hline Casing media & $\begin{array}{c}\text { Phenolics }(\mathrm{mg} / \mathrm{g} \\
\text { dray weight) } \\
(\text { Mean } \pm \text { SE) }\end{array}$ & $\begin{array}{c}\text { Flavonoids(mg/g } \\
\text { dray weight) } \\
\text { (Mean } \pm \text { SE) }\end{array}$ & $\begin{array}{l}\text { Antioxidant }(\%) \\
\quad(\text { Mean } \pm \text { SE })\end{array}$ \\
\hline $100 \%$ Peat moss (Control) & $237.10 \pm 4.80^{\mathrm{a}}$ & $51.36 \pm 0.71^{\mathrm{b}}$ & $72.88 \pm 0.57^{\mathrm{a}}$ \\
\hline $75 \%$ Peat moss $+25 \%$ Spent mushroom & $218.47 \pm 9.47^{\mathrm{a}}$ & $53.32 \pm 0.79^{\mathrm{b}}$ & $58.06 \pm 0.88^{\mathrm{e}}$ \\
\hline $75 \%$ Peat moss $+25 \%$ Vermi & $218.60 \pm 7.07^{\mathrm{a}}$ & $52.37 \pm 1.53^{\mathrm{b}}$ & $70.13 \pm 1.00^{b}$ \\
\hline $\begin{array}{l}50 \% \text { Peat moss }+25 \% \text { Spent mushroom }+ \\
25 \% \text { Vermi }\end{array}$ & $223.53 \pm 0.43^{\mathrm{a}}$ & $50.39 \pm 1.39^{b}$ & $67.58 \pm 0.29^{c}$ \\
\hline $50 \%$ Peat moss $+50 \%$ fronds & $227.20 \pm 3.67^{\mathrm{a}}$ & $57.21 \pm 0.95^{\mathrm{a}}$ & $72.22 \pm 0.32^{\mathrm{a}}$ \\
\hline $\begin{array}{l}50 \% \text { Peat moss }+25 \% \text { fronds }+25 \% \\
\text { Vermi }\end{array}$ & $222.70 \pm 9.90^{\mathrm{a}}$ & $51.62 \pm 0.95^{\mathrm{b}}$ & $50.20 \pm 1.18^{\mathrm{f}}$ \\
\hline F value & 1.316 & 9.907 & 111.537 \\
\hline P value & 0.313 & 0.000 & 0.000 \\
\hline
\end{tabular}

Means with different letters in the same columns are significantly different $(\mathrm{P}<0.05)$ by Duncan multiple test

Table 10: Effect of casing materials of A. bisporus (S-2) strain for phenolics, flavonoids and antioxidant.

\begin{tabular}{|c|c|c|c|}
\hline Casing media & $\begin{array}{c}\text { Phenolics }(\mathrm{mg} / \mathrm{g} \\
\text { dray weight) } \\
\text { (Mean } \pm \text { SE) }\end{array}$ & $\begin{array}{c}\text { Flavonoids(mg/g } \\
\text { dray weight) } \\
\text { (Mean } \pm \text { SE) } \\
\end{array}$ & $\begin{array}{c}\text { Antioxidant } \\
(\text { Mean } \pm \text { SE })(\%)\end{array}$ \\
\hline $100 \%$ Peat moss (Control) & $243.03 \pm 2.40^{\mathrm{ab}}$ & $54.79 \pm 1.24^{\mathrm{a}}$ & $69.47 \pm 0.82^{\mathrm{a}}$ \\
\hline $75 \%$ Peat moss $+25 \%$ Spent mushroom & $208.70 \pm 1.82^{\mathrm{c}}$ & $46.81 \pm 1.04^{\mathrm{c}}$ & $42.80 \pm 0.90^{\mathrm{d}}$ \\
\hline $75 \%$ Peat moss $+25 \%$ Vermi & $252.93 \pm 9.10^{\mathrm{a}}$ & $52.53 \pm 0.38^{\mathrm{ab}}$ & $54.09 \pm 1.06^{\mathrm{c}}$ \\
\hline $\begin{array}{l}50 \% \text { Peat moss }+25 \% \text { Spent mushroom }+ \\
25 \% \text { Vermi }\end{array}$ & $244.03 \pm 4.40^{\mathrm{ab}}$ & $51.56 \pm 0.60^{\mathrm{b}}$ & $60.48 \pm 0.76^{\mathrm{b}}$ \\
\hline $50 \%$ Peat moss $+50 \%$ fronds & $219.47 \pm 9.14^{\mathrm{c}}$ & $53.97 \pm 0.90^{\mathrm{ab}}$ & $52.05 \pm 1.02^{\mathrm{c}}$ \\
\hline $\begin{array}{l}50 \% \text { Peat moss }+25 \% \text { fronds }+25 \% \\
\text { Vermi }\end{array}$ & $209.00 \pm 6.14^{\mathrm{c}}$ & $47.91 \pm 1.46^{\mathrm{c}}$ & $43.08 \pm 0.94^{\mathrm{d}}$ \\
\hline F value & 6.360 & 10.482 & 121.236 \\
\hline P value & 0.002 & 0.000 & 0.000 \\
\hline
\end{tabular}

Means with different letters in the same columns are significantly different $(\mathrm{P}<0.05)$ by Duncan multiple test 


\section{Conclusion}

In the present study, six different casing layers were tested which prepared locally from available wastes for enhancing production of two strains of white button mushroom Agaricus bisporus (S-1 and S-2) and evaluated pharmaceutical substances content. Tested casing layers were $100 \%$ peat moss (control), peat moss + spent mushroom (3:1), peat moss + vermicompost (3:1), peat moss + spent mushroom + palm fronds $(2: 1: 1)$, peat moss + palm fronds $(1: 1)$ and peat moss + vermicompost + palm fronds (2:1:1). All casing layers gave high mushroom production as well as high contents of total phenol, flavonoids and antioxidant activity. The results showed that, casing layer peat moss + palm fronds + vermicompost was the best layer for production and harvest quality of the two strains.

\section{References}

Ahlawat, O.P., 2002. Fluctuations in $\mathrm{pH}$ and temperature in the compost and compost casing interface their effect on yield of Agaricus bisporus. Mush. Res., 11(1):11-14.

Bhatt, P., K.P.S. Kushwaha and R.P. Singh, 2006. Physicochemical properties of different casing mixtures and its effect on yield of Agaricus bisporus. Mush. Res. 15(1): 29-32.

Bors, W., and M. Saran, 1987. Radical scavenging by flavonoid antioxidants. Free Radical Research and Communications, 2: 289-294.

Chandra, R., V.N. Pandey, and M.H.B. Singh, 2014. Comparative evaluation of casing mixtures on the yield potential of button mushroom (Agaricus bisporus). Proceedings of the ${ }^{8}$ th International Conference on Mushroom Biology and Mushroom Products (ICMBMP8) pg. 345-350.

Cheung, L.M., P.C.K. Cheung, and V.E.C. Ooi, 2003. Antioxidant activity and total phenolics of edible mushroom extracts. Food Chem., 81(2):249-255.

Choudhary, D., 2011. First Preliminary report on isolation and characterization of novel Acinetobacter spp. in casing soil used for cultivation of button mushroom, Agaricus bisporus (Lange) Imbach. International Journal of Microbiology.790285.

Dhar, B.L., O.P. Ahlawat, G. Pradeep and R. Dev, 2006. Casing layer as related to mushroom yield and quality in Agaricus bisporus in India. Mush. Res., 15(2): 111-117.

Eldeeb, A., 2017. Recycling Agricultural Waste as a Part of Interior Design and Architectural History in Egypt. The Academic Research Community Publication, 1(1):1-7.

Ergun, B., N. Osman, M. Colak and T. Hilmi, 2007. Cultivation of Agaricus bisporus on some compost formulas and locally available casing materials. Part I: Wheat straw based compost formulas and locally available casing materials. African J. of Biotechnology , 6 (19): 2225-2230.

Gerrits, J.P.G., 1974. Development of a synthetic compost for mushroom growing based on wheat straw and chicken manure. Netherlands J. of Agricultural Science, 22: 175-194.

Halliwell, B., 1997. Antioxidants in human health and disease. Annual Reviews of Nutrition, 16: $33-$ 50 .

Harman, D., 1997. Role of free radical reactions in aging and disease. Journal of Geriatric Dermatology, 5: 114-127.

Ishaq, M., M. Fiaz, M. Saif ullah, and M. Sharait Ullah, 2017. Evaluation of mycelial growth of Oyster mushroom (Pleurotus floridanus Singer) on different media and cereal grains. Journal of Biodiversity and Environmental Sciences, 11 (3): 67-72.

Kapoor, J.N., 2004. Mushroom Cultivation. Department of Mycology and Plant Pathology, IARI, New Delhi, 14-15.

Kaur, A.P. and V.K. Rampal 2017. Assessment of Casing Mixtures on Yield Potential and Quality of Button Mushroom (Agaricus bisporus) - On Farm Trial. Int. J. Curr. Microbiol. App. Sci., 6(2): 430-436.

Kaur, A., P.P. Johl, and H.S. Sodhi, 2017. Evaluation of casing variables for cultivation of Agaricus bisporus strain U3 in Punjab, India. Journal of Applied and Natural Science, 9 (1): $305-310$.

Kohli, M., 1984. Judging when to pick an mushroom: An aid to picker. Mushroom J., 138: 207-209.

Kumar N., S.K. Biswas, K. Lal, D. Baboo and A. Hussain, 2019. Integrated effect of different substrates on growth parameters and yield of Pleurotus sajor caju. J. Mycopathol. Res., 57(1): 35-40. 
Kumar, B., C. Kumari, and M. Kumar, 2018. Effect of bio-fertilizers on mycelial growth and physical properties of white button mushroom, Agaricus bisporus. Int. J. Curr. Microbiol. App. Sci., 7(2): 2216-2222.

Kumar, K., S.K. Biswas, A. Husain, and S. Kumar, 2020. Physical parameters of casing materials on yield parameter of white button mushroom [Agaricus bisporus (longe. Imboch)]. Int. J. Chem. Stud., 8(1):2820-2824.

Kumarasamy, Y., M. Byres, P.J. Cox, M. Jasapars, L. Nahar and S.D. Sarker, 2007. Screening seeds of some Scottish plants for free-radical scavenging activity. Phytother Res., 21: 615-621.

Lo, K.M. and P.C.K. Cheung, 2005. Antioxidant activity of extracts from the fruiting bodies of Agrocybe aegerita var. alba. Food Chemistry, 89: 533-539.

Mami, Y., G. Peyvast, M. Ghasemnezhad, and F. Ziaie, 2013. Supplementation at casing to improve yield and quality of white button mushroom. Agricultural Sciences, 4: 27-33.

Mantel, E.F.K., R.K. Agrawal and P.K. Seth 1972. A guide to mushroom cultivation. Ministry of Agriculture, Farm Information Unit, Directorate of Extension, New Delhi, Farm Bull. No.2.

Quettier, D.C., B. Gresseier, J. Vasseur, T. Dine, C. Brunet and M.C. Luyckx, 2000. Phenolic compounds and antioxidant activities of buckwheat (Fagopyrum esculentum Moench) hulls and flour. J. Ethnopharmacol., 72: 35-42.

Ram, R.C., and S.K. Holkar, 2009. Bio-efficacy of casing materials for growth stages, physical parameters and yield potential of Agaricus bisporus. Mush. Res., 18 (2):65-68.

Shrivastava, N., 2012. Casing layer as related to mushroom yield and quality of Agaricus bisporus in India. Proceedings of 18th International Society for Mushroom Science (ISMS) Congress, August 26-30, 2012 at Beijing, China, August 26-30.

Singh, M., R.P. Singh, and H.S. Chaube, 2000. Impact of physiochemical properties of casing on yield of Agaricus bisporus (Lange) Imbach. (Van. Griensven, ed.) In: Science and cultivation of Edible fungi Balkema, Rotterdam, 441-446.

Singleton, V.L., R. Orthofer and R.M. Lamuela-Raventós, 1999. Analysis of total phenols and other oxidation substrates and antioxidants by means of Folin-Ciocalteu reagent. Methods in Enzymology, 299: 152-178.

Skerget M., P. Kotnik, M. Hadolin, A.R. Hras, M. Simonic and Z. Knez, 2005. Phenols, proanthocyanidins, flavones and flavonols in some plant materials and their antioxidant activities. Food Chemistry, 89: 191-198.

Sonnenberg, A.S., J.J. Baars, W. Gao, and R.G. Visser 2017. Developments in breeding of Agaricus bisporus var. bisporus: progress made and technical and legal hurdles to take .Appl. Microbiol. Biot., 101: 1819-29.

Tekao, T., N. Watanabe, I. Yagi and K. Sakata, 1994. A simple screening method for antioxidant and isolation of several antioxidants produced by marine bacteria from fish and shellfish. Biosci. Biotechnol. Biochem., 58: 1780-1783.

Yadav, M.K., R. Chandra, S.K. Yadav, S.K. Yadav, P.K. Dhakad, A.K. Srivastava, P.K. Dwivedi and S. Naik, 2017. Comparative evaluation of locally available casing materials for quantitative and qualitative effect on two strains of Agaricus bisporus (Lange). Biochem. Cell. Arch., 17(1): 133-139. 